\title{
Totally thoracoscopic repair of atrial septal defect reduces systemic inflammatory reaction and myocardial damage in initial patients
}

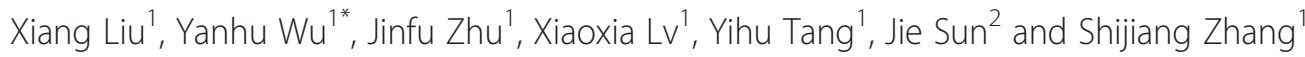

\begin{abstract}
Background: To compare the effect of totally thoracoscopic with conventional, open repair of atrial septal defect.

Methods: Forty atrial septal defect cases were divided into two groups by surgical approach: totally thoracoscopic approach (group $A, n=20$ ) and conventional open approach (group $B, n=20$ ). In group $A$, surgical procedures were performed through three portal incisions in the right lateral chest wall under thoracoscopic vision without the aid of a computerized robotic surgical system. Notably, all operations were completed by one surgeon who had just begun using this technique. In group B, the atrial septal defects were repaired in conventional open fashion. Clinical outcomes and serum levels of tumor necrosis factor a (TNF-a), interleukin-6 (IL-6), interleukin-10 (IL-10), intercellular adhesion molecule 1 (ICAM-1), and creatine kinase isoenzyme-myocardial band (CK-MB) for the two groups were evaluated and compared.
\end{abstract}

Results: All operations were performed successfully without serious complications. Durations of cardiopulmonary bypass (CPB), CPB setup, aortic cross-clamping, and operative procedure were significantly longer in group A than in group $B(P<0.05)$. The recovery times for body temperature and laboratory values of leukocytes were significantly shorter for group A than for group B $(P<0.05)$. There were no differences in durations of postoperative assisted ventilation or intensive care unit and hospital stays, volumes of blood transfused intraoperatively or thoracic drainage, or medical costs between the two groups. Serum levels of inflammatory factors (TNF-a, IL-6, IL-10, and ICAM-1) and CK-MB increased significantly in both groups after surgery. However, $6 \mathrm{~h}$ and $12 \mathrm{~h}$ after surgery, levels of these inflammatory factors and CK-MB were significantly lower in group $A$ than in group $B(P<0.05)$.

Conclusions: Thoracoscopic cardiac surgery is technically feasible and safe, with less trauma and quicker recovery even when done by a surgeon newly introduced to the technique.

Keywords: Total thoracoscopy, Atrial septal defect, Cardiac surgery, Systemic inflammatory response, Myocardial damage

\section{Background}

Repair of atrial septal defect (ASD) using a totally thoracoscopic approach is the oldest and most popular application of video-assisted thoracoscopic cardiac surgery [1-3]. Since 2008, we have carried out ASD repair using a totally thoracoscopic approach with satisfactory clinic results and without the aid of a computerized robotic surgical system. However, thoracoscopic cardiac surgery

\footnotetext{
* Correspondence: wuyanhu@njmu.edu.cn

'Department of Cardiothoracic Surgery, First Affiliated Hospital of Nanjing

Medical University, Nanjing, China

Full list of author information is available at the end of the article
}

is still controversial because its minimally invasive nature makes the surgery longer and more difficult, especially because of the newness of this technique. In the present study, to evaluate the advantages of this method when performed by a surgeon newly introduced to this technique, we compared the outcomes of thoracoscopic cardiac surgery in 20 consecutive patients with those of conventional thoracotomy with regard to postoperative systemic inflammatory response syndrome (SIRS) and myocardial injury.

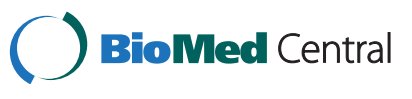

(C) 2014 Liu et al.; licensee BioMed Central Ltd. This is an Open Access article distributed under the terms of the Creative Commons Attribution License (http://creativecommons.org/licenses/by/2.0), which permits unrestricted use, distribution, and reproduction in any medium, provided the original work is properly credited. The Creative Commons Public Domain Dedication waiver (http://creativecommons.org/publicdomain/zero/1.0/) applies to the data made available in this article, unless otherwise stated. 


\section{Methods}

\section{Patients and surgeon}

Forty patients from our hospital who were diagnosed with ASD and underwent repair between 2010 and 2012 were divided into two groups: totally thoracoscopic (group A, $\mathrm{n}=20$ ) and conventional open (group $B, \mathrm{n}=20$ ) surgical approaches. Echocardiograms demonstrated ASD without other cardiovascular anomalies. Only patients who could not undergo percutaneous interventional closure because of defect size or ASD type were included. Various factors including age, sex, weight, cardiothoracic ratio, cardiac function as demonstrated by ejection fraction, size of ASD, and hematocrit were compared. There were no significant differences in these parameters between groups (Table 1). Both methods were considered feasible in the hospital. Patient consent was not deemed necessary by the local research and ethics committee (Nanjing Medical University Institutional Review Board).

Thoracoscopic cardiac surgeries were performed by one surgeon newly introduced to this technique. The surgeon's preoperative preparation included attaining a large amount of theoretical knowledge of endoscopy and carrying out a great deal of endoscopic, especially thoracoscopic, training; mastery of routine and minimally invasive cardiac surgeries and demonstrating the ability to deal with unexpected situations independently; mastery of thoracic surgical skill and ability to be trained in total thoracoscopic lung lobectomy; practice performing the techniques on pigs; and observation of totally thoracoscopic cardiac surgery at other hospitals.

\section{ASD surgical repair}

The patients in group A were placed under endotracheal anesthesia via a double-lumen endotracheal tube and the femoral artery and vein were cannulated. Left single-lung ventilation was applied. Oxygen saturation was monitored. If oxygen saturation fell below $90 \%$, double-lung ventilation was initiated. A 2-cm-long incision was made in the fifth intercostal space at the right midaxillary line. A trocar was placed through the incision and the thoracoscope (Stryker Inc., Kalamazoo, MI, USA) was inserted through

\section{Table 1 Patient baseline characteristics}

\begin{tabular}{lccc}
\hline Variables & $\begin{array}{c}\text { Group A } \\
(\mathbf{n = 2 0})\end{array}$ & $\begin{array}{c}\text { Group B } \\
(\mathbf{n = 2 0})\end{array}$ & $\begin{array}{c}\boldsymbol{P} \\
\text { value }\end{array}$ \\
\hline Age (years, mean \pm 1SD) & $22.13 \pm 2.2$ & $27.95 \pm 2.3$ & 0.073 \\
Gender (male) & $9(45 \%)$ & $6(30 \%)$ & 0.327 \\
Weight (kg, mean \pm 1 SD) & $51.13 \pm 12.2$ & $51.43 \pm 8.8$ & 0.932 \\
Cardiothoracic ratio (\%, mean \pm 1 SD) & $52 \pm 4.9$ & $53.55 \pm 3.6$ & 0.281 \\
EF (\%, mean \pm 1 SD) & $65.53 \pm 4.1$ & $68.61 \pm 5.2$ & 0.067 \\
Size of ASD (mm, mean \pm 1 SD) & $29.87 \pm 4.73$ & $26.83 \pm 7.42$ & 0.182 \\
Hematocrit (\%, mean \pm 1 SD) & $0.39 \pm 0.04$ & $0.39 \pm 0.03$ & 0.816 \\
\hline
\end{tabular}

$A S D$, atrial septal defect; $E F$, ejection fraction; $S D$, standard deviation. the trocar. Another 2-cm incision was made parasternally at the third intercostal space for insertion of surgical instruments and the inferior vena cava snare. A third 2-cm incision was made in the second intercostal space at the right mid-axillary line for insertion of surgical instruments, the superior vena cava snare, a cannula for cold perfusion, and an aortic clamp (Figure 1). For all intrathoracic procedures, the surgical field was displayed on a screen and a computerized robotic surgical system was not employed. The ASD was closed with direct running suture and in some cases reinforced with a Dacron patch. Group B patients underwent with single-lumen endotracheal anesthesia and conventional cardiopulmonary bypass (CPB). Patients were positioned supine for access to the mid-sternal surgical area.

All patients were anesthetized using fentanyl 10-20 $\mu \mathrm{g} / \mathrm{kg}$, midazolam $0.1-0.15 \mathrm{mg} / \mathrm{kg}$, isoflurane $0.2-1.5 \%$, and/or propofol $50-100 \mu \mathrm{g} / \mathrm{kg} / \mathrm{min}$. Muscle relaxation was achieved with rocuronium $0.6-1.0 \mathrm{mg} / \mathrm{kg}$. Heparin was given to maintain an activated clotting time (ACT) $>420 \mathrm{~s}$ during $\mathrm{CPB}$. Bypass management included membrane oxygenation (Affinity NT; Medtronic Inc., Fridley, MN, USA) and maintenance of mean arterial pressure between 55 and $85 \mathrm{mmHg}$, temperature between 32 and $35^{\circ} \mathrm{C}$, and blood sugar between 4 and $10 \mathrm{mmol} / \mathrm{L}$. Myocardial protection was achieved with cold blood crystalloid cardioplegia, and terminal warm-blood cardioplegic reperfusion (a hot-shot) of 250-500 mL was delivered just prior to the removal of the aortic cross-clamp. After the patient came off $\mathrm{CPB}$, heparin was reversed with protamine (approximately $10 \mathrm{mg} / 1,000$ units of heparin). Postoperatively, patients were managed in a specialized cardiovascular intensive care unit with standardized protocols for early extubation and blood glucose control (target 5.1 to $8.0 \mathrm{mmol} / \mathrm{L})$.

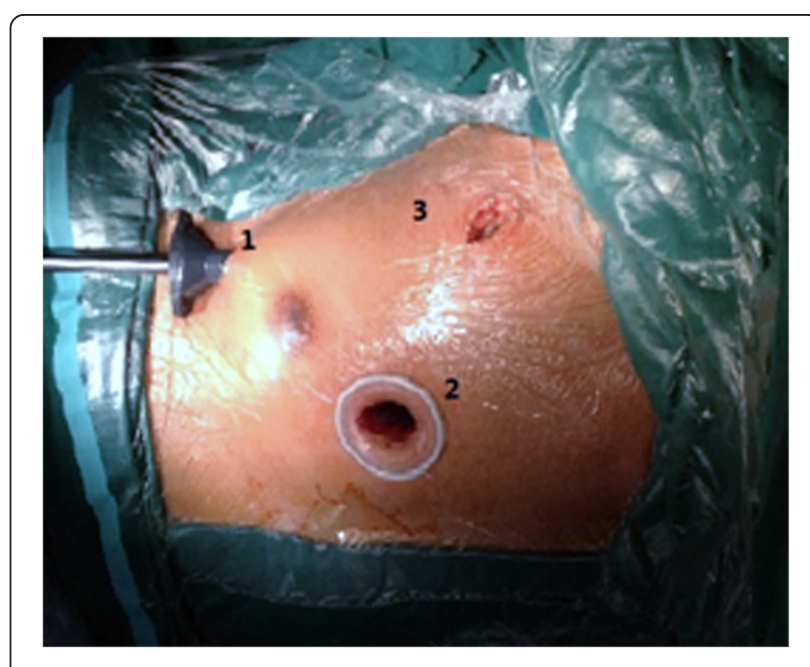

Figure 1 Surgical incisions and portal sites. 


\section{Clinical outcome measures}

Volumes of blood transfused and thoracic drainage; durations of $\mathrm{CPB}$ setup, $\mathrm{CPB}$, aortic cross-clamping, the operative procedure, postoperative assisted ventilation, and ICU and hospital stays; and medical costs were recorded for both groups.

\section{Blood sampling and measurement}

Whole venous blood $(10 \mathrm{~mL})$ was taken from all patients preoperatively; immediately after aortic opening; and 2, $6,12,24$, and $72 \mathrm{~h}$ postoperatively. The samples were centrifuged for $10 \mathrm{~min}$ at 3,200 rpm, and the supernatants were collected and preserved at $-80^{\circ} \mathrm{C}$. Levels of tumor necrosis factor- $\alpha$ (TNF- $\alpha$ ), interleukin-6 (IL-6), intercellular adhesion molecule-1 (ICAM-1), interleukin10 (IL-10), and creatine kinase isoenzyme-myocardial band (CK-MB) were measured by enzyme-linked immunosorbent assay (ELISA) using commercially available ELISA kits (ADL Biotech Inc., Mashteuiatsh, QC, Canada). All tests on samples and standards were performed in duplicate per manufacturer instructions.

\section{Statistical analysis}

Statistical analysis was performed using SPSS for Windows, Version 13.0 (SPSS Inc., Chicago, IL, USA). All variables are presented as mean \pm standard deviation (SD). Patient baseline characteristics (except sex, which was compared using the $\chi^{2}$ test) and operative results were compared with independent $t$-tests. One-way analysis of variance was used to analyze serum levels of inflammatory factors and CK-MB. A value of $P<0.05$ was considered statistically significant.

\section{Results}

All operations were performed successfully without serious complications. Patients in both groups were cured and were followed for a mean of 24 months (range, 18 to 34 months). All patients could return to work and activities of daily living and had no long-term complications.

The recovery times for body temperature and laboratory values of leukocytes were significantly shorter for group A than for group B (Table 2). There were no differences between the two groups in durations of postoperative assisted ventilation or ICU and hospital stays; no differences in volumes of blood transfused or thoracic drainage, and no difference in medical costs (Table 2). Durations of $\mathrm{CPB}, \mathrm{CPB}$ setup, ascending aortic cross-clamping, and operation were significantly longer in group $A$ than in group B (Table 2).

Serum levels of inflammatory factors (TNF- $\alpha$, IL-6, ICAM-1, IL-10) and CK-MB did not differ significantly between groups before $\mathrm{CPB}$ but significantly increased after CPB.

TNF- $\alpha$ levels peaked $6 \mathrm{~h}$ after aortic opening in both groups. Values for group $\mathrm{B}$ were higher than those for group $\mathrm{A}$ at each time point of $\mathrm{CPB}$ and differed significantly at $0 \mathrm{~h}$ (immediately after aortic opening) and 6, 12 , and $24 \mathrm{~h}$ after aortic opening (Figure 2).

IL-6 levels peaked $6 \mathrm{~h}$ after aortic opening in both groups. Group B IL-6 values were greater than those for group $\mathrm{A}$ at each time point of CPB and differed significantly at 2 and $12 \mathrm{~h}$ after aortic opening (Figure 3 ).

Levels of ICAM-1 peaked $2 \mathrm{~h}$ after aortic opening in both groups. ICAM-1 values were greater in group B than in group $\mathrm{A}$ at each $\mathrm{CPB}$ time point and were significantly different at 2, 6, and $24 \mathrm{~h}$ after aortic opening (Figure 4).

Table 2 Operative results

\begin{tabular}{lccc}
\hline Variables (mean \pm 1 SD) & $\begin{array}{c}\text { Group A } \\
(\mathbf{n = 2 0 )}\end{array}$ & $\begin{array}{c}\text { Group B } \\
(\mathbf{n = 2 0})\end{array}$ & $52.45 \pm 9.9$ \\
value
\end{tabular}

$C P B$, cardiopulmonary bypass; ICU, intensive care unit; $R M B$, Renminbi (Chinese currency); $S D$, standard deviation. 


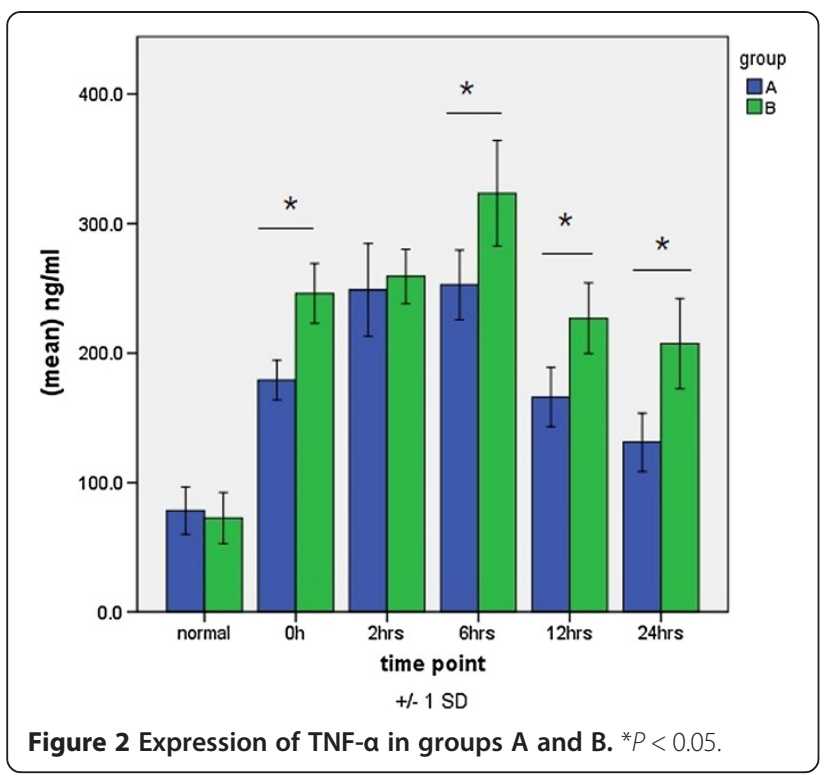

IL-10 levels of IL-10 peaked $12 \mathrm{~h}$ after aortic opening in both groups, with a value significantly higher in group $\mathrm{B}$ than in group A $12 \mathrm{~h}$ after aortic opening (Figure 5).

$\mathrm{CK}-\mathrm{MB}$ level peaked $6 \mathrm{~h}$ after aortic opening in group $\mathrm{A}$ and $24 \mathrm{~h}$ after aortic opening in group $\mathrm{B}$, with values greater in group $B$ than in group $A$ at each $C P B$ time point and significant differences seen 6,24 , and $72 \mathrm{~h}$ after aortic opening (Figure 6).

\section{Discussion}

One of the current arguments against totally thoracoscopic cardiac surgery is that it does not comply with the requirements of minimal invasion because the durations of $\mathrm{CPB}$ and ascending aortic cross-clamping associated

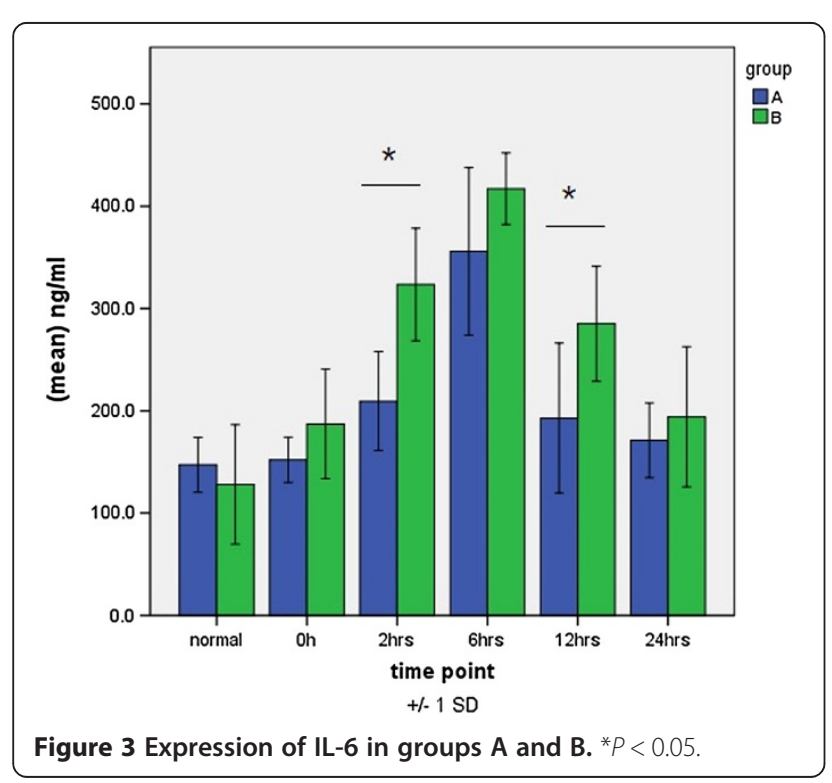

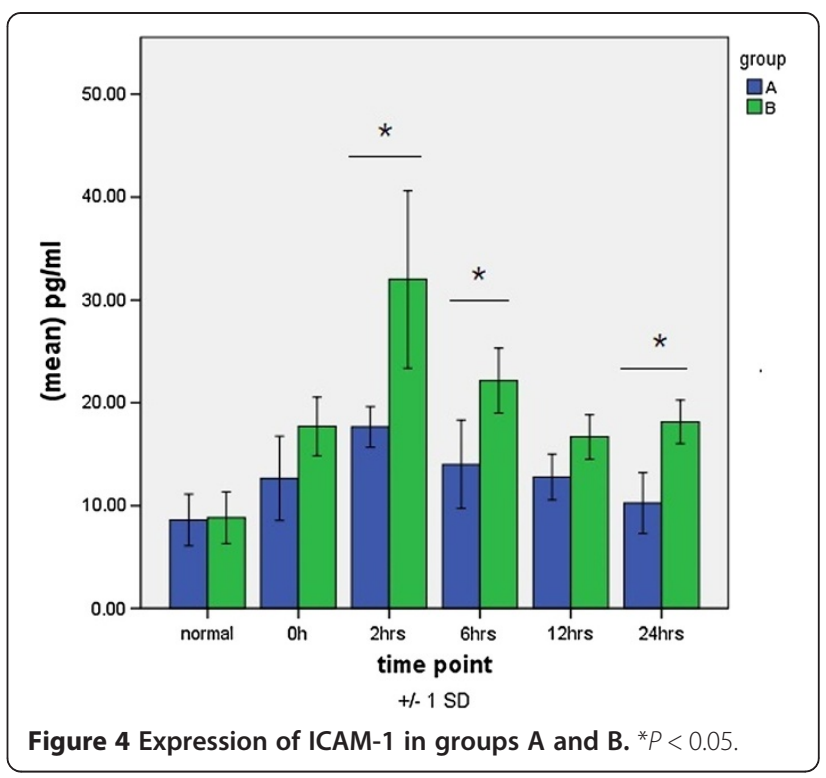

with this method are significantly prolonged [4-6]. Open ASD repair is usually completed by a junior surgeon, and a senior surgeon often acts as instructor and assistant. Therefore, operative duration, including CPB and aortic cross-clamping, were longer in group B. Nevertheless, durations of $\mathrm{CPB}, \mathrm{CPB}$ setup, aortic cross-clamping, and operation in group $B$ were significantly shorter than in group A. The corresponding activation of SIRS after CPB in group A should be more serious and may cause severe impairment of organ function; thus, because of potential trauma to the patient, the thoracoscopic technique does not meet the claim of minimal invasion. Especially since this work had just begun, durations of $\mathrm{CPB}, \mathrm{CPB}$ setup, ascending aortic cross-clamping, and operation in group

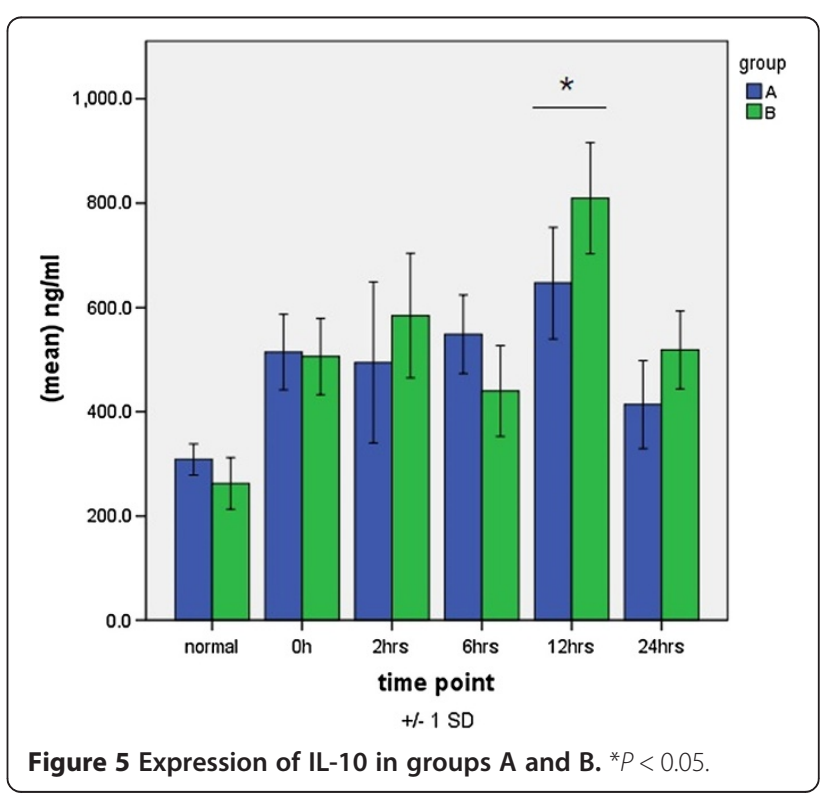




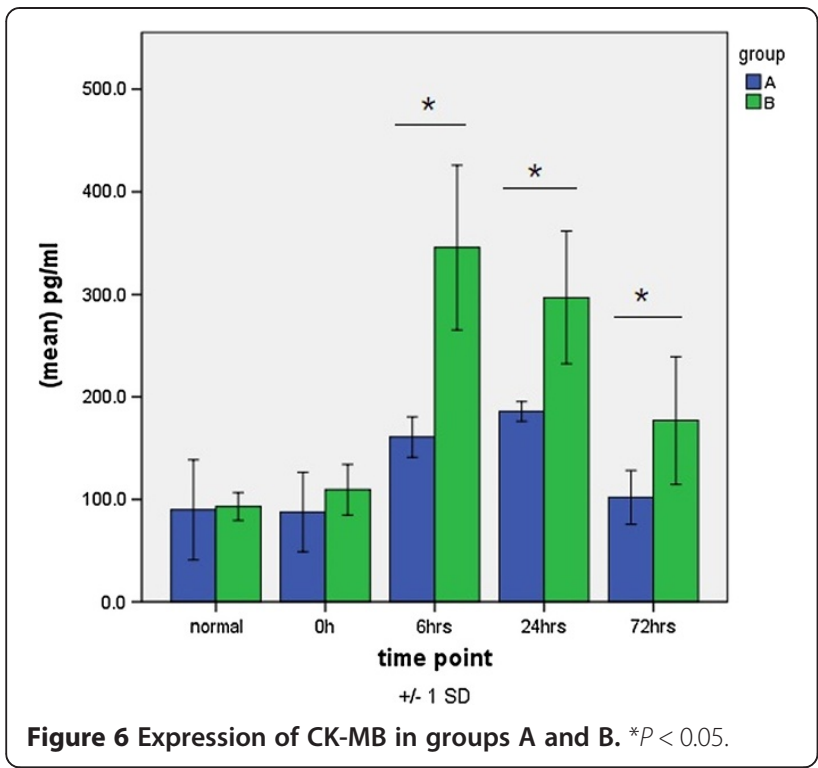

A were not only significantly longer than in group B, but also longer than in thoracoscopic cardiac surgical procedures completed by a skilled and experienced doctor [3,7].

SIRS occurs after CPB mainly because of the contact of blood with foreign body surfaces during CPB. This leads to multi-system and multi-cell activation and produces a large number of humoral and cellular inflammatory mediators through a cascade reaction, which eventually causes tissue and organ damage or even multiple organ dysfunction syndrome [8-10]. Causes of SIRS also include surgical injury, anesthesia, changes in body temperature, organ ischemia/reperfusion injury, machine blood transfusion, hemodilution, and heparin-protamine complexes $[11,12]$. It is important to understand the claim of minimal invasion of totally thoracoscopic cardiac surgery apart from the size of the incision.

Levels of TNF- $\alpha$, IL-6, and ICAM-1, which are the main inflammatory cytokines occurring after $\mathrm{CPB}$ and which play critical roles in the inflammatory cascade, are important in evaluating SIRS after CPB. They are released first and are also the most important endogenous mediators in the inflammatory process after CPB [13-15]. In the present study, we found that in patients who underwent totally thoracoscopicASD repair, levels of TNF- $\alpha$, IL-6, and ICAM-1 increased early on and were significantly higher than the increased values induced by $\mathrm{CPB}$, aortic cross-clamping, and other operation. However, in patients who underwent thoracoscopicASD repair, elevations in the levels of TNF- $\alpha$, IL- 6 , and ICAM-1 were of short duration, dropped soon after operation, and were significantly lower than those in patients who underwent open surgery at the same period. Our finding suggests that the degree of SIRS activated by totally thoracoscopicASD repair is lower than that in conventional surgery if other factors, such as recovery from surgical trauma and postoperative cardiopulmonary function, are included.

The prognosis of SIRS after CPB also depends on another important factor, the balance between proinflammatory factors and anti-inflammatory factors. IL-10 is an important post-CPB anti-inflammatory factor that has an endogenous protective effect that counters tissue and cell destruction caused by inflammatory mediators $[15,16]$. However, if the anti-inflammatory response to SIRS is too strong, it will reduce immune function and increase the body's susceptibility to infection, and induce or aggravate multiple organ failure. The compensatory anti-inflammatory response syndrome (CARS) is a complex pattern of immunologic responses to severe infection or injury. Many studies have shown that the timing and relative magnitude of these responses have a profound impact on patients' outcomes $[17,18]$. Our study suggests that the level of the anti-inflammatory cytokine IL-10 in totally thoracoscopicASD repair maintains good homeostasis with levels of inflammatory cytokines, reaches a peak in the early postoperative period, and drops quickly. The peak value is also lower than that in conventional surgery, which indirectly reflects the smaller systemic inflammatory response of thoracoscopic cardiac surgery.

CK-MB level in serum increases when there is myocardial injury. It is widely used to determine perioperative myocardial injury after cardiac surgery and is an important indicator of the recovery of cardiac function and prognostic risk factors $[19,20]$. The present study has shown that the extent of myocardial injury after thoracoscopicASD repair was significantly lower than after conventional surgery, which is a prerequisite for the successful rehabilitation of patients who undergo cardiac surgery.

The main reasons for SIRS and myocardial injury are $\mathrm{CPB}$ and surgical injury. Compared with conventional thoracotomy, thoracoscopic cardiac surgery avoids a large chest incision and sternotomy wound, significantly reduces tissue damage, and maintains the overall structure of the thorax. In addition, the blunt trauma of endoscopic surgery, such as stretching and squeezing the heart, is greatly decreased. Thus, thoracoscopic cardiac surgery may prevent SIRS and myocardial injury.

\section{Conclusions}

Although it is more difficult and dangerous, after careful preparation, thoracoscopic cardiac surgery is technically feasible and creates less trauma and quicker recovery. In addition, thoracoscopicASD repair, which can be characterized as minimally invasive, results in a reduced systemic inflammatory response after $\mathrm{CPB}$, maintains the balance of proinflammatory and anti-inflammation factors, reduces tissue and organ damage, even when performed by a surgeon new to this technique. 


\section{Abbreviations}

ASD: Atrial septal defect; CARS: Compensatory anti-inflammatory response syndrome; CK-MB: Creatine kinase isoenzyme-myocardial band; CPB: Cardiopulmonary bypass; ELISA: Enzyme-linked immunosorbent assay; ICAM-1: Intercellular adhesion molecule-1; ICU: Intensive care unit; IL-6: Interleukin-6; IL-10: Interleukin-10; RMB: Renminbi (unit of Chinese currency); SD: Standard deviation; SIRS: Systemic inflammatory response syndrome; TNF-a: Tumor necrosis factor-a.

\section{Competing interests}

The authors declare that they have no competing interests.

\section{Authors' contributions}

YHW conceived and designed the experiments. $X \mathrm{~L}$ analyzed the data and wrote the manuscript. XXL and YHT performed blood sampling and the experiments. SJZ supervised the study, participated in its design and coordination, and drafted the manuscript. JFZ and JS advised on clinical implications. All authors read and approved the final manuscript.

\section{Acknowledgments}

The authors are indebted to the patients who kindly agreed to take part in this study. They are also grateful to the cardiovascular surgeons involved with the study for providing patient blood samples pre- and postoperatively. Finally, the authors thank First Affiliated Hospital of Nanjing Medical University for its financial support.

\section{Author details}

${ }^{1}$ Department of Cardiothoracic Surgery, First Affiliated Hospital of Nanjing Medical University, Nanjing, China. ${ }^{2}$ Department of Cardiothoracic Anesthesiology, First Affiliated Hospital of Nanjing Medical University, Nanjing, China.

Received: 18 October 2013 Accepted: 21 February 2014 Published: 11 March 2014

\section{References}

1. Chen Q, Cao H, Zhang GC, Chen LW, Chen DZ: Safety and feasibility of intra-operative device closure of atrial septal defect with transthoracic minimal invasion. Eur J Cardiothorac Surg 2012, 41:121-125.

2. Yu SQ, Cai ZJ, Cheng YG, Duan DW, Xu XZ, Chen WS, Zhou GX: Video-assisted thoracoscopic surgery for congenital heart disease. Asian Cardiovasc Thorac Ann 2002, 10:228-230.

3. Ma ZS, Dong MF, Yin QY, Feng ZY, Wang LX: Totally thoracoscopic closure for atrial septal defect on perfused beating hearts. Eur I Cardiothorac Surg 2012, 41:1316-1319.

4. Stevens LM, Rodriguez E, Lehr EJ, Kindell LC, Nifong LW, Ferguson TB, Chitwood WR Jr: Impact of timing and surgical approach on outcomes after mitral valve regurgitation operations. Ann Thorac Surg 2012, 93:1462-1468

5. Andrade WL, Amoretti JR: Minimally invasive surgical valve repair. Heart Surg Forum 2010, 13:E165-E167.

6. Murzi M, Kallushi E, Solinas M, Glauber M: Video-assisted right atrial surgery with a single two-stage femoral venous cannula. Interact Cardiovasc Thorac Surg 2009, 9:9-10

7. Cheng Y, Chen H, Mohl W, Liu X, Si Z: Totally endoscopic congenital heart surgery compared with the traditional heart operation in children. Wien Klin Wochenschr 2013, 125:704-708.

8. Sitniakowsky LS, Later AF, van de Watering LM, Bogaerts M, Brand A, Klautz RJ, Smit NP, van Hilten JA: The effect of RBC transfusions on cytokine gene expression after cardiac surgery in patients developing post-operative multiple organ failure. Transfus Med 2011, 21:236-246.

9. Jouan J, Golmard L, Benhamouda N, Durrleman N, Golmard JL, Ceccaldi R, Trinquart L, Fabiani JN, Tartour E, Jeunemaitre X, Menasche P: Gene polymorphisms and cytokine plasma levels as predictive factors of complications after cardiopulmonary bypass. J Thorac Cardiovasc Surg 2012, 144:467-473.

10. Machado LB, Negri EM, Bonafé WW, Santos LM, SáMalbouisson LM, Carmona MJ: Evaluation of cytokine levels and pulmonary function in patients undergoing coronary artery bypass graft. Rev Bras Anestesiol 2011, 61:275-285
11. Francischetti I, Moreno JB, Scholz M, Yoshida WB: Leukocytes and the inflammatory response in ischemia-reperfusion injury. Rev Bras Cir Cardiovasc 2010, 25:575-584.

12. Heyn J, Beiras-Fernandez A, Luchting B, Briegel J, Weis F: Inflammatory reactions and hydrocortisone in the setting of cardiac surgery: an overview. Cardiovasc Hematol Agents Med Chem 2011, 9:56-61.

13. Jungwirth B, Eckel B, Blobner M, Kellermann K, Kochs EF, Mackensen GB: The impact of cardiopulmonary bypass on systemic interleukin- 6 release, cerebral nuclear factor-kappa B expression, and neurocognitive outcome in rats. Anesth Analg 2010, 110:312-320.

14. Shu $L$, Wei XC: Effect of Penehyclidine hydrochloride in systemic inflammatory response syndrome caused by cardiopulmonary bypass. Sichuan Da Xue Xue Bao Yi Xue Ban 2012, 43:543-546.

15. Liou HL, Shih CC, Chao YF, Lin NT, Lai ST, Wang SH, Chen HI: Inflammatory response to colloids compared to crystalloid priming in cardiac surgery patients with cardiopulmonary bypass. Chin J Physiol 2012, 55:210-218.

16. Sipos W, Duvigneau C, Sterz F, Weihs W, Krizanac D, Bayegan K, Graf A, Hartl R, Janata A, Holzer M, Behringer W: Changes in interleukin-10 mRNA expression are predictive for 9-day survival of pigs in an emergency preservation and resuscitation model. Resuscitation 2010, 81:603-608.

17. Gentile LF, Cuenca AG, Efron PA, Ang D, Bihorac A, McKinley BA, Moldawer LL, Moore FA: Persistent inflammation and immunosuppression: a common syndrome and new horizon for surgical intensive care. J Trauma Acute Care Surg 2012, 72:1491-1501.

18. Mayerle J, Dummer A, Sendler M, Malla SR, van den Brandt C, Teller S, Aghdassi A, Nitsche C, Lerch MM: Differential roles of inflammatory cells in pancreatitis. J Gastroenterol Hepatol 2012, 27(Suppl 2):47-51.

19. Ceyhan D, Tanriverdi B, Bilir A: Comparison of the effects of sevoflurane and isoflurane on myocardial protection in coronary bypass surgery. Anadolu Kardiyol Derg 2011, 11:257-262.

20. Nair S, Iqbal K, Phadke M, Jadhav UE, Khandekar J, Khandeparkar JM: Effect of cardiopulmonary bypass on tissue injury markers and endothelial activation during coronary artery bypass graft surgery. $J$ Postgrad Med 2012, 58:8-13.

doi:10.1186/2047-783X-19-13

Cite this article as: Liu et al: Totally thoracoscopic repair of atrial septal defect reduces systemic inflammatory reaction and myocardial damage in initial patients. European Journal of Medical Research 2014 19:13.

\section{Submit your next manuscript to BioMed Central and take full advantage of:}

- Convenient online submission

- Thorough peer review

- No space constraints or color figure charges

- Immediate publication on acceptance

- Inclusion in PubMed, CAS, Scopus and Google Scholar

- Research which is freely available for redistribution 